\title{
Study for Femto Satellites Using Micro Control Moment Gyroscope
}

\author{
Mark Post \\ Space Mechatronic Systems Technology Laboratory \\ Department of Design, Manufacture and Engineering Management, University of Strathclyde \\ Glasgow, United Kingdom \\ mark.post@strath.ac.uk \\ Ralf Bauer \\ Centre for Microsystems Photonics \\ Department of Electronic Electrical Engineering, University of Strathclyde \\ Glasgow, United Kingdom \\ ralf.bauer@strath.ac.uk \\ Regina Lee \\ Department of Earth and Space Science and Engineering, York University \\ Toronto, Canada \\ reginal@yorku.ca \\ Junquan Li \\ Clyde Space Ltd \\ Glasgow, United Kingdom \\ junquan.li@clyde-space.com
}

Abstract-Femto-satellites can be used for distributed space missions that can require hundreds to thousands of satellites for real time, distributed, multi-point networks to accomplish remote sensing and science objectives. While suitable sensors are available using micro-electro-mechanical system technology, most femto-satellite designs have no attitude control capability due to the power and size constraints on attitude control actuators. A novel femto-satellite design that uses a micro-electromechanical system Control Moment Gyroscope is studied in this paper. We focus on the principal design, modelling, and discussion of the proposed Control Moment Gyroscope while detailing a controllable femto-satellite design that can make use of attitude control for simple sensing missions.

\section{TABle of Contents}

1 INTRODUCTION $. . . \ldots \ldots \ldots \ldots \ldots \ldots \ldots \ldots \ldots \ldots, 1$

2 Proposed Femto-SATellite System Design 2

3 Proposed MEMS CMG DESIGN ........... 3

4 Simulation Results and Discussion ...... 5

5 Conclusions ......................... 6

ACKNOWLEDGMENTS $. \ldots \ldots \ldots \ldots \ldots \ldots \ldots \ldots . .6$

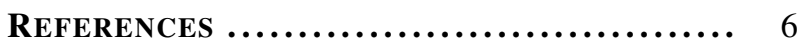

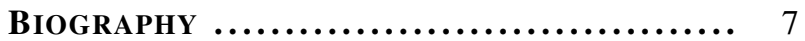

\section{INTRODUCTION}

A distributed space system is a system of multiple spacecraft designed to work in a coordinated group to perform a mission, such as communications, navigation, remote sensing, space exploration and science. A distributed spacecraft system using femto-satellites can provide significant flexibility and robustness compared to a large satellite [1]. Due to the mass, size and power constraints of femto-satellites, very few Attitude and Orbit Control System (AOCS) options are available [2], and the tolerances of micro AOCS systems can be very limited. Next generation sensors for femto-satellites have been studied for use in disaster management missions [3]. Femto-satellites can be based on CMOS (Complementary Metal-Oxide Semiconductor) and MEMS (Micro Electro

978-1-4799-5380-6/15/\$31.00 (c)2015 IEEE.

Mechanical System) technologies [4], but few active control systems have been proposed for femto-satellites. The Control Moment Gyroscope is a space attitude control actuator which acts as torque amplifier [15]. It is more efficient than a reaction wheel in terms of power consumption and slew rate [16]. A MEMS control moment gyroscope for a sub kilogram spacecraft has been studied [17], but no feasibility study for such a MEMS CMG for femto-satellites has been done. The power requirements, thermal design, and performance capabilities of MEMS actuator systems are heavily dependent on environmental variables such as temperature variation, and study of the on-orbit operational requirements for a femtosatellite is a vital part of the design process. We propose the use of a MEMS CMG and conduct a feasibility study for a femto-satellite attitude control system in this paper to illustrate the potential capabilities and key challenges for such a mission.

\section{Femto Satellite Survey}

Ref. [1] proposed a swarm of $100 \mathrm{~g}$ wafer-scale femtosatellite with 6 DOF (Degree of Freedom) for the distributed spacecraft system. The current MEMS reaction wheels can provide $10^{-9}-10^{-10} \mathrm{Nm}$ torque. Ref. [1] also states that Commercial Off-The-Shelf (COTS) cell phone vibration motors can provide $10^{-5} \mathrm{Nm}$ torque. A MEMS propulsion system can be used for orbit control of femto-satellites, but attitude control is the focus of our proposed femto-satellite. Refs. [2], [3] and [4] discuss the system design, sensors and applications of femto-satellites. PCBsat $(70 \mathrm{~g})$ and Wikisat $(19.7 \mathrm{~g})$ are two existing femto-satellites [4] that include a CMOS imager as a payload. PCBsat has two sun sensors, a GPS receiver, and passive aerodynamic attitude control [4]. Wikisat has four optic sensors, a 3D accelerometer, a 3D gyrometer and magnetorquers for attitude control [4]. Ref. [5] compared the system requirements of femto-satellites for formation flying, constellation, fractionated spacecraft and swarms. For an Earth observation oriented mission, the femto-satellite swarms can be used to measure the sea surface, cyclone detection, nowcasting, and Earth gravity modelling. Ref. [6] studied the methods for building a femto-satellite mission with COTS. Ref. [7] studied a femtosatellite system design and mini launcher. Another challenge for such a tiny femto-satellite is the communication system [8], as the directivity of the antenna and the accuracy to point 
ground stations or other femto-satellite in the swarm must be increased.

\section{Control Moment Gyroscope Survey}

Future science and exploration missions will strongly rely on innovative Guidance Navigation and Control (GNC) technologies. The CMG has been also used for space robotics, underwater vehicles, and on the International Space Station as well as for other large space missions [13] and[14], but Control Moment Gyroscopes are not often considered for use on small satellites [9], [10], [11] and [12]. The main reasons are due to the complexity of the mechanical and control system needed to implement an effective CMG. CMG systems have many advantages over reaction wheels and magnetic rods, such as a large torque amplification capability, large moment storage capability, and high angular agility. By applying advances in computer aided design tools and electronics technologies, $\mathrm{CMG}$ systems have more recently been considered for small satellites [15] and [16]. It also has been considered for femto-satellites [17] and [18]. The main challenges with the creation of a MEMS CMG are the silicon micro machining technology and the control system [23].

\section{MEMS Actuator Survey}

The future science and exploration missions will be critically dependent on the development, validation and infusion of micro-electro-mechanical systems (MEMS) based spacecraft guidance navigation and control avionics they are highly integrated, power efficient, and minimally packaged which can be flexible to meet specific mission requirements [19], [20], [21], [22] and [23]. For MEMS actuators, some additional considerations should be included in order to get the best functionality, such as pull-in-instability and high $\mathrm{Q}$ factors. [24]. The space environment poses many environmental hazards, and not all materials are suitable for that environment. Electronic components can exhibit unpredictable behaviours without shielding, thermal gradients are wide, and the temperature changes fast and beyond the usual ranges inside the atmosphere. MEMS sensors and actuator functionality have to be tested with thermal, vibration and radiation testing [25] and [26] before being used for femto-satellite missions.

\section{Proposed Femto-Satellite System DESIGN}

Figures 1 and 2 show a high-level concept design for the proposed femto-satellite and its power and actuator drive systems. The proposed femto-satellite has three surfaces joined at right angles in a half-cube configuration. Each surface covers the outer side of the half-cube with solar panels and is intended to remain sun-pointing by attitude control assisted by solar radiation pressure. The inner side holds the electrical power system, battery, data handling microcontroller (OnBoard Computer), watchdog timer, communication system (radio and transceiver), attitude and orbit determination and control system, thermal system, and a selected payload (considered to be a CMOS imager) [2].

A dedicated electronic controller and high-voltage drive circuit is necessary for micro-electro-mechanical system (MEMS) electrostatic actuators. Different MEMS actuators have specific requirements for their power supplies $(10-$ $300 v)$, activation current ( $1 \mu \mathrm{A}$ to $5 \mathrm{~mA})$ and commutation speed [27]. The controller of the proposed MEMS CMG will initially be based on a programmable micro-controller core and in more developed versions, Field-Programmable Gate

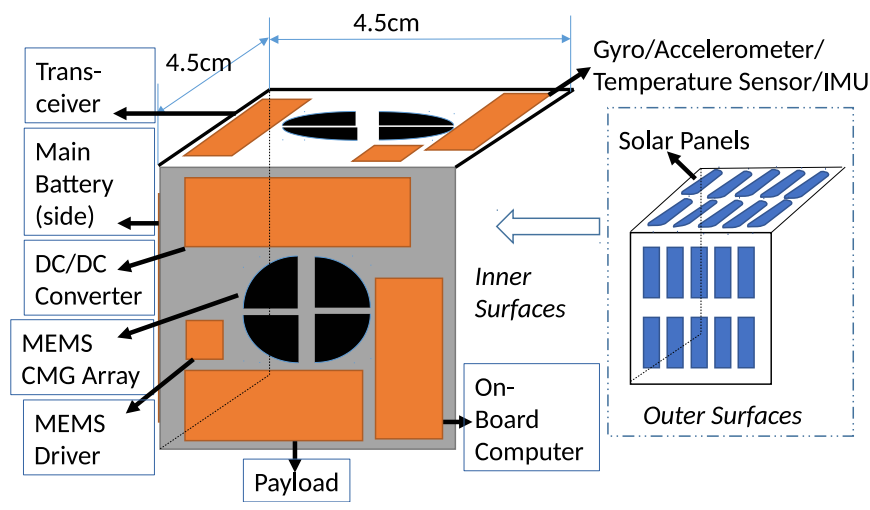

Figure 1. Femto-Satellite Structure with MEMS CMG

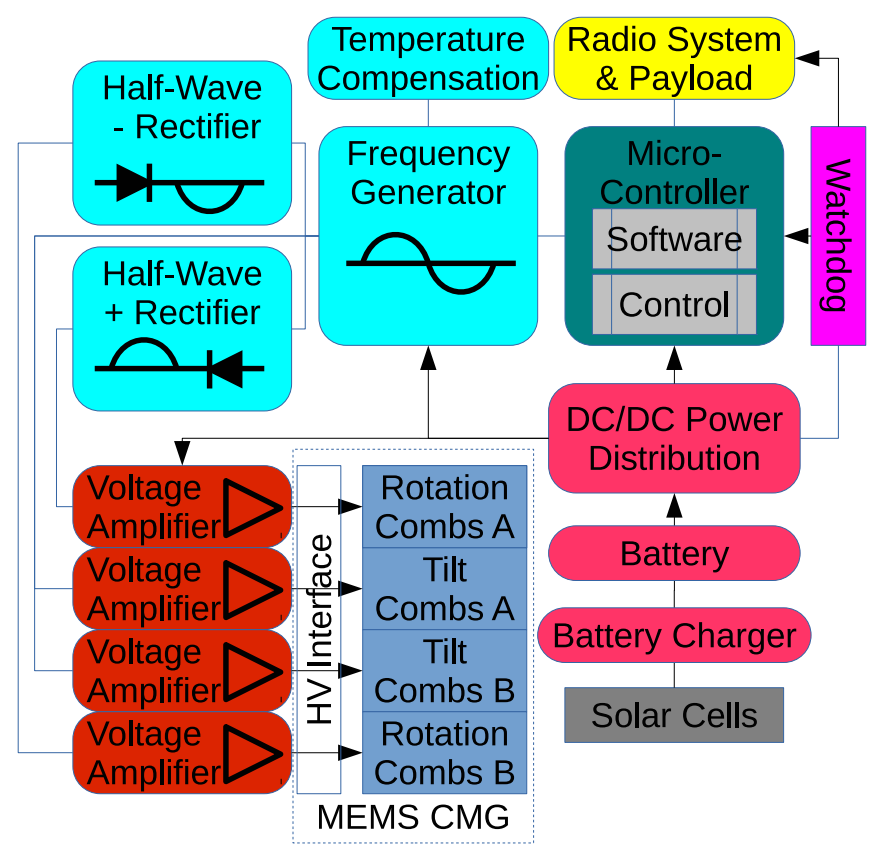

Figure 2. Power and Drive System Design for Femtosatellite with MEMS CMG

Array (FPGA) technologies. Very Large Scale Integration (VLSI) of power and control components directly on the MEMS CMG is being studied, but at present a separate semiconductor circuit is required for housing the control electronics of the device to simplify single-process manufacture of the CMG. A micro-PCB that forms a side of the femtosatellite will contain the circuitry, and a microcontroller will generate the control signals to the High-Voltage (HV) drive electronics that connect to the MEMS electrostatic loads and set the output voltage of the DC/DC controller that feeds the MEMS CMG. Due to the high Q factor of the MEMS CMG components, precise control of drive frequencies is necessary, and a temperature compensation circuit is used to stabilize the drive frequency over wide temperature ranges. Only very small adjustments of the torque output can be achieved by frequency and voltage control, so the attitude control algorithm creates large changes in torque by simply switching the CMG on and off at appropriate rates by means of a low-frequency pulse-width modulated (PWM) signalling scheme. 


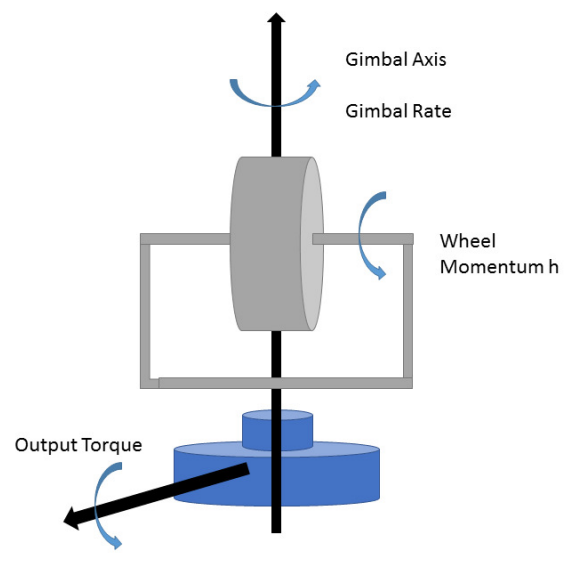

Figure 3. Principle of CMG.

\section{Proposed MEMS CMG DESIGN}

\section{CMG Principle}

The working principle of the MEMS CMG is similar to a single gimbal CMG (shown in Figure 3). In order to get the equations of the torque output, two reference frames, gimbal reference frame $\left(x_{g}, y_{g}\right.$ and $\left.z_{g}\right)$ and substrate reference frame $\left(x_{s}, y_{s}\right.$ and $\left.z_{s}\right)$ are defined. When the gimbal angles are zero, the two frames coincide with each other. The spinning rotor is assumed as a rigid body which is symmetric about the spinning axis $z_{g}$ in the gimbal frame. The rotor rotates with an angular velocity $\omega$. Therefore the angular momentum of the rotor in the substrate frame is defined as

$$
h=J \omega
$$

where $J$ is the inertial moment of the spinning rotor about $z_{g}$.

The gimbal rate is defined as $\dot{D}_{\delta}$. The CMG output torque is perpendicular to the directions of $\omega$ and $\dot{D}_{\delta}$.

$$
T=h \dot{D_{\delta}}
$$

In the proposed MEMS CMG, the angular sinusoidal vibrations substitute for the angular rotations. The angular displacements of the rotor and gimbal can be written as

$$
\begin{aligned}
& D_{r}=A_{r} \sin (2 \pi f t) \\
& D_{\delta}=A_{g} \sin (2 \pi f t)
\end{aligned}
$$

where $A_{r}$ and $A_{g}$ are the angular vibration amplitude of the rotor and the gimbal, respectively, and $f$ is the resonant frequency of the rotor and gimbal. The angular velocities of the rotor and gimbal can be written as

$$
\begin{gathered}
\omega=2 \pi f A_{r} \cos (2 \pi f t) \\
\dot{D}_{\delta}=2 \pi f A_{g} \cos (2 \pi f t)
\end{gathered}
$$

Substituting Equations 1-6, the output torque can be written as

$$
T=J(2 \pi f)^{2} A_{r} A_{g} \cos ^{2}(2 \pi f t)
$$

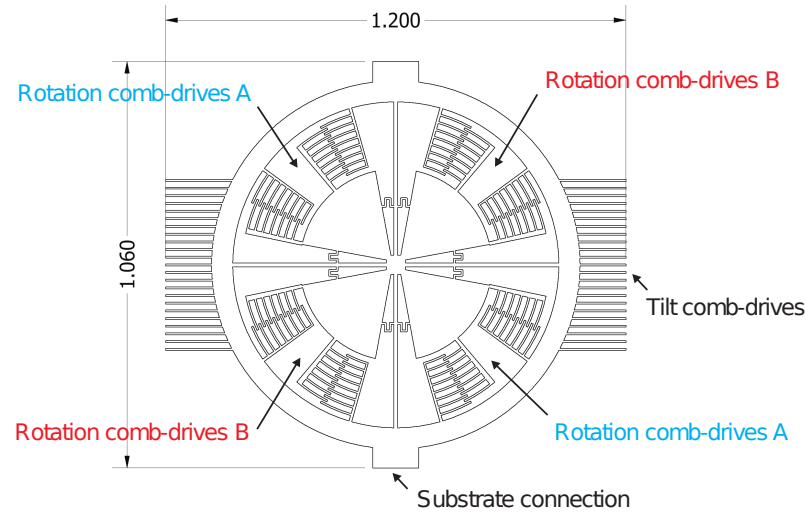

Figure 4. Proposed design for the single device layer MEMS CMG, showing the moving parts of the CMG and highlighting the comb-drive actuators which would require the three different drive signals to create the combined rotational and tilt oscillation for creating the torque output of the CMG.

The amplitude of the MEMS CMG output torque is proportional to the inertial moment of the spinning rotor, resonant frequency and the amplitude of vibration.

\section{Design of MEMS CMG}

The design of the proposed MEMS CMG is influenced by a targeted fabrication possibility using a cost-effective and reliable multi-user single crystal silicon-on-insulator (SOI) process. The proposed design consists of a single device layer and is based on a rotational oscillation of a disk which is coupled to a frame with a tilt oscillation about its main axis. This main axis is defined by the substrate connections of the fully released moving structure. Both the rotational and the tilt oscillation are coupled by using resonance movement modes of the same frequency. This, combined with the choice of an appropriate phase relationship between the two movements, allows for the generation of a uni-directional torque which can be reversed by applying a $180^{\circ}$ phase shift between the electrical actuation signals of both movements.

The actuation principle of the proposed MEMS CMG is based on the use of capacitive electrostatic comb-drives for both movement modes. Electrostatic actuation exhibits general advantages of lower power consumption compared to other MEMS actuation mechanisms, with the specific case of comb-drive actuators reducing the risk of pull-in phenomena and requiring in general less actuation voltages compared to backside electrode actuators. Additionally, rotational combdrive actuated tilt devices have shown the ability to generate tilt angles of $80^{\circ}$ for $8-10 \mathrm{kHz}$ movements [32] even in atmospheric pressure conditions. The single device layer employed by the proposed use of a multi-user process allows for easy comb alignment without the necessity to employ additional wafer bonding or other functional parts, as would be the case for electromagnetic actuation or backside capacitive electrodes.

The proposed MEMS CMG design is shown in Figure 4, only depicting the moving part of the device with the substrate and counterpart of the outer comb fingers omitted for clarity. The design has a four-segment inner rotating disk of $500 \mu \mathrm{m}$ diameter, connected via four $10 \mu \mathrm{m}$ by $400 \mu \mathrm{m}$ rectangular beams to an outer frame of $480 \mu \mathrm{m}$ outer diameter. Each rotating disk segment has 2 banks of 7 combs to create the rotational oscillation, with 2 segments each contributing 
the rotation force for each direction of rotation. Combdrives $\mathrm{A}$ allow in this case for a clock-wise actuation, with comb-drives B being responsible for the counter-clockwise actuation. The design limits the maximum rotation angle of the oscillation to $\pm 9^{\circ}$. The $55 \mu \mathrm{m}$ wide outer frame anchors the fixed combs of the rotational comb-drives, the moving combs for the tilt movement and the connection to the torsional spring connection to the silicon substrate. Not shown in the schematic of Figure 4 is the necessary isolation between the fixed rotation comb-drives and the rest of the structure. This can be done using an additional fabrication process step or through a post-process coating step creating a solid connection between physically separated parts of the MEMS CMG structure.

The proposed CMG design was chosen with the use of a multi-user fabrication process in mind. This will allow the use of a cost-effective and well established process which will allow quick turn around with proven process stability. The envisaged multi-user fabrication process is the single crystal silicon-on-insulator fabrication process SOIMUMPs offered by Memscap Inc. [28]. The process is based on a phosphors doped, $25 \mu \mathrm{m}$ SOI device layer, using a single etch step of this device layer to create the functional device parts and a further backside etch step to fully release the functional parts from a $400 \mu \mathrm{m}$ thick silicon handle waver. The doped device layer will allow for electrical connectivity of the actuators. Each fabricated MEMS chip consists of a $9 \times 9 \mathrm{~mm}^{2}$ design area which would allow for the creation of an array of 9-12 synchronized MEMS CMG's per chip.

Figure 5 shows the system model of the MEMS CMG. As shown in Figure 2, the power electronics for the MEMS CMG are designed around a simple frequency generator, which drives both the rotation and gimbal tilt of the CMG synchronously. The comb drive used for tilting the gimbal plate of the CMG around two twisting beams causes the movable combs to vibrate about the horizontal, where the outer combs are placed. Therefore, at a given frequency both the positive and negative parts of the full-wave cycle can be used for the tilt combs. The rotating wheel of the CMG is actuated by a push-pull arrangement of combs, and constrained to the plane of vibration by four rotor beams. The two sets of rotation combs are driven alternately, so half-wave rectifiers are used to split the positive and negative parts of the cycle. Separate voltage ladders are then used to raise the voltage for each of the four drive outputs on the power circuit, which are connected through a high-voltage interface on the MEMS die.

\section{Rotor Calculation of MEMS CMG}

If the comb-drive is actuated by a single-sided voltage, the force exerted by each movable comb finger ([29]) can be approximated by

$$
F=\frac{\epsilon h V^{2}}{g}
$$

The total torque generated by the entire rotary comb-drive is given by

$$
T=\frac{\epsilon h V^{2}}{g} N R
$$

$N$ is the number of comb fingers, $R$ is the radius of each comb finger, $\epsilon$ is the permittivity of the single crystal silicon devicelayer, $h$ is the high of the comb fingers, $V$ is the voltage difference between the moving combs and the fixed combs, and $g$ is the horizontal distance between the comb fingers.

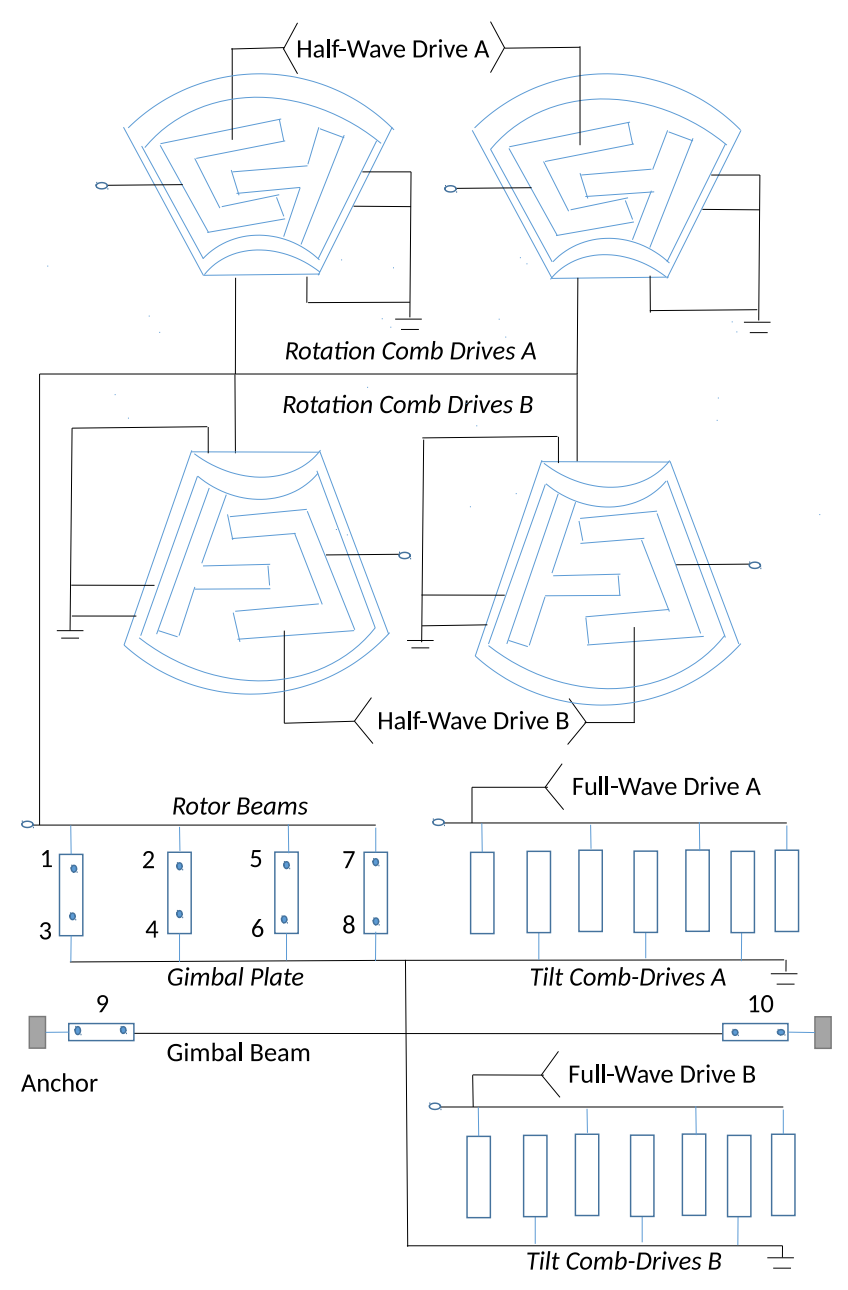

Figure 5. System Level Model of MEMS CMG.

\section{Gimbal Calculation of MEMS CMG}

The movement of the gimbal is created by applying a singleor dual-sided voltage signal to the comb-drive electrodes located on the substrate of the MEMS chip. This resonant movement is governed by the equation of motion for a forced oscillator, with the drive force of the oscillator being given by:

$$
F=\frac{1}{2} V^{2}(t) \frac{\partial C}{\partial \theta}
$$

where $C$ is the capacitance of the gimbal comb-drive and $\theta$ is the tilt angle of the gimbal around its major axis.

The calculation of the capacitance distribution with varying angular displacements of the comb-pairs of the comb-drive can be done using 2D finite element modelling (FEM) simulation to account for any fringe field effects between the comb electrodes [32]. The resulting movement is then additionally influenced by the damping and spring constants of the MEMS structure. 


\section{Simulation Results and Discussion}

For the electro-mechanical simulation of the MEMS CMG the finite element modelling (FEM) software COMSOL Multiphysics is used, looking at the mechanical resonance eigenfrequencies of the proposed CMG and later on toward a full electromechanical simulation. The material properties of the single crystal silicon of the envisaged SOIMUMPs process have been investigated before [31], with their anisotropic parameters being used as basis for the FEM simulation. The first two mechanical oscillation eigenmodes of the proposed MEMS CMG are shown in Figure 6, depicting the tilt movement around the major axis of the device at $55.1 \mathrm{kHz}$ and the rotation movement of the inner disk at $55.2 \mathrm{kHz}$. By synchronizing both movements through appropriate electrical actuation signal control the combined movement would result in the synchronous switching of both the rotation and tilt movement directions, therefore creating a resulting torque in a single direction.

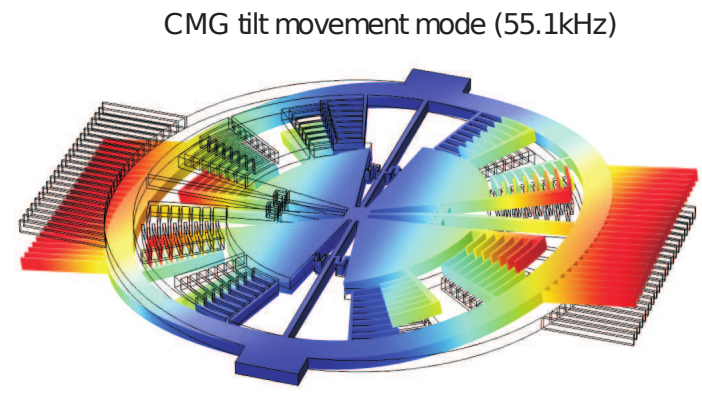

CMG rotational movement mode $(55.2 \mathrm{kHz})$

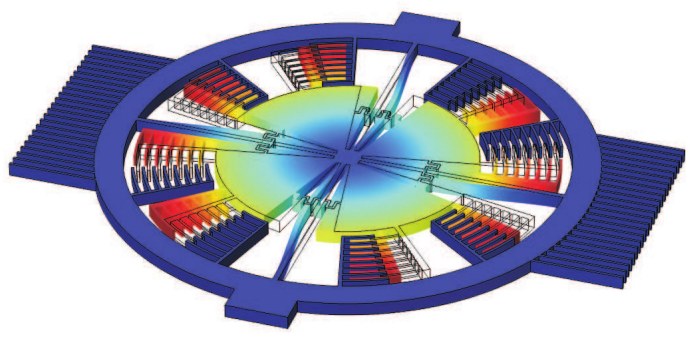

Figure 6. Simulated eigenmode shapes of the proposed MEMS CMG, showing the rotational oscillation at $55.2 \mathrm{kHz}$ and the tilt oscillation at $55.1 \mathrm{kHz}$.

To estimate the potential torque that can be generated by the MEMS CMG, equation 7 is used with the design parameters of the proposed device. The mass moment of inertia $J$ for the rotating part of the CMG is calculated as $4.7 \cdot 10^{-16} \mathrm{~m}^{2} \mathrm{~kg}$, using the same FEM simulation as for the simulation of the device resonance frequencies, with the frequency $f$ of both the rotation and tilt movements being $55 \mathrm{kHz}$. For an assumed rotation amplitude of $9^{\circ}$ and a tilt amplitude of $10^{\circ}$ this leads to a maximum torque for a single MEMS device approximated as $1.5 \cdot 10^{-6} \mathrm{Nm}$. This maximum torque occurs during the centre crossing of the synchronized rotational and tilt oscillation. This torque is almost 2 orders of magnitude higher than the value estimated by [17], which can be mainly attributed to the increase in the oscillation frequency for the here proposed design.

\section{Discussion of Thermal Effects}

As the MEMS CMG has not yet been fabricated, definite testing of its thermal and radiation characteristics at this stage is not possible. In particular, radiation testing is very time- consuming and costly due to the facilities required. However, we can attempt to estimate its performance and limitations based on prior work that has been done on similar comb-drive devices.

The main influence that temperature is expected to have on the MEMS CMG is the temperature dependent Youngs modulus of Silicon, which determines the resonance characteristics of the CMG. Many commercial MEMS comb-drive devices can tolerate the approximately $-65^{\circ} \mathrm{C}$ to $120^{\circ} \mathrm{C}$ temperature range in low Earth orbit without critical failure, and the highest chance of failure in MEMS devices is due instead to launch vibration [37], the accuracy of operation of the device often suffers. Temperature sensitivity on the resonant frequency is in the range of $31 \mathrm{ppm} /{ }^{\circ} \mathrm{C}$ close to room temperature [31], but over a temperature variation on the order of $100^{\circ} \mathrm{C}$, this can often be up to the range of $1 \%$ of the resonance frequency. The $\mathrm{CMG}$ is intended to be driven synchronously as close to its resonance frequency as possible to minimize the effect of changes in the high $\mathrm{Q}$ factor of the comb drive with temperature [35], but this requires that the resonance frequency be either constrained or tracked.

One way to reduce temperature influences on resonant MEMS structures is to use highly-doped Silicon for the structure, as heavy doping can change the temperature dependence of elastic constants in silicon by on the order of $100 \mathrm{ppm} /{ }^{\circ} \mathrm{C}$ [33]. This approach can also cause residual stresses and other unwanted effects in the semiconductor though, and needs further research before becoming a viable option. It is also possible to adapt the design dimensions of a MEMS device by means of a simulation optimization loop so that a flat capacitance response with temperature is generated as used by Liu et al. [34], but a significant amount of design and optimization effort is needed, and further simulation work is needed to evaluate the feasibility of this approach for the proposed MEMS CMG layout.

Active temperature compensation has also been developed for MEMS devices. Prikhodko, Trusov, and Shkel propose self-referencing of the temperature for compensation of a MEMS Quadruple Mass Gyroscope through accurate sensing of the drive-mode resonance frequency with a phase-locked loop that monitors frequency shifts relative to a high-stability frequency reference [36]. For a high Q-factor system such as this, a $10 p p b$ frequency resolution was possible near room temperature, and reduction of temperature-induced drift from approximately $500^{\circ} / \mathrm{hr}$ to under $2^{\circ} / \mathrm{hr}$. Even trading off the high precision of measurement of this system for a wider temperature range compensation, we expect that a similar compensation system can be used to accurately track changes in CMG resonance and allow the CMG to continue operation across low Earth orbit temperature ranges.

\section{Discussion of Radiation Effects}

Degradation of MEMS devices due to radiation is generally due to two mechanisms: atomic displacement from collisions, and ionization energy of extra electron-hole pairs [38]. While displacement damage can lead to permanent degradation of semiconductor devices due to changes in carrier quantity and carrier mobility, it is expected to have less impact on a MEMS structure that does not rely on minority carrier balance for function, and it is additionally stated that the physical damage to silicon and dielectric insulators in terms of material properties is rather small. The high sensitivity of devices such as resonators to small changes in material properties may be affected to some degree, but electrical and ionization-based failure modes are in general much more 
likely to affect MEMS devices.

Ionization damage can have a major impact on the function of low-voltage VLSI MOS ICs, and can cause failure of electrostatically-actuated devices through trapped charge in the structure. The potential of trapped charge to affect MEMS devices is significant, but depends on the layout and actuation voltage of the device, as several comb-drive devices have exhibited changes in dielectric charging of the device up to $50 \mathrm{krad}$ of proton radiation but no permanent functional damage, and a conducting polysilicon film was shown to prevent such degradation up to $3 M \mathrm{rad}$ [39]. Several years in low earth orbit would be needed before appreciable changes would be observed, and greater care would be needed in the design of the drive and control circuitry than the MEMS gyro itself.

Lozano, Palumbo and Alurralde describe a MEMS microrelay device with a $20 \mu m$ thick single crystal silicon layer, thirty $3 \mu \mathrm{m}$ width and $3 \mu \mathrm{m}$ gap comb drive fingers, and an activation voltage from $22 \mathrm{~V}-50 \mathrm{~V}$ [40]. When exposed to a $10 \mathrm{MeV}$ proton beam, capacitance between the contact pad of the comb drive and the P-type silicon changed only on the order of $0.05 \%$ with total doses between $1.7 e 9 p+/ \mathrm{cm}^{2}$ and $3.5 e 11 p+/ \mathrm{cm}^{2}$, and at constant capacitance a shift in voltage of approximately $-4.5 \mathrm{~V}$. This did not cause a significant variation in a nominal activation voltage of $23 \mathrm{~V}$, the level of leakage current between the pads of the structure, and the conduction resistance of the device before and after irradiation. Although it is expected that the shift in voltage at constant capacitance will change the activation voltage to some degree, the observations are that very little shift is present. Given the relatively high voltages required to drive the $\mathrm{CMG}$, this shift is not expected to significantly impact performance.

\section{Conclusions}

We have discussed in this paper a feasible femto-satellite design featuring attitude control that makes use of a MEMSbased attitude determination and control system. While small, this femto-satellite contains all the fundamental components for a controllable satellite, and can be capable of formation flying and simple measurements. The MEMS CMG is designed both for efficient operation, and for reliable and cost-effective fabrication using multi-user processes. Future work will include more detailed design and simulation for both the MEMS CMG and femto-satellite model, which will culminate in the fabrication of a functioning MEMS CMG system.

\section{ACKNOWLEDGMENTS}

The authors would like to thank Prof. Deepak Uttamchandani at the University of Strathclyde for his assistance with this research.

\section{REFERENCES}

[1] Fred Y. Hadegh, Soon-Jo Chung and Harish M. Manohara, "On Development of 100 Gram Class Spacecraft for Swarm Applications", IEEE System Journal, Vol. 99, No. 99, 2014.

[2] David J. Barnhart, Tanya Vladimirova, Adam M Baker, Martin, N Sweeting, "A Low Cost Femtosatellite to
Enable Distributed Space Missions", Acta Astronautica, Vol. 64, pp 1123-1143, 2009.

[3] Luis Izquierdo, Joshua Tristancho, "Next Generation of Sensors for Femto Satellite Based on Commercial of the Shelf", IEEE/AIAA, Digital Avionics System Conference, 2011.

[4] Nizar Tahri, Chaffa Hamrouni, Adel M. Alimi, "Study of Current Femto Satellite Approaches and Services", International Journal of Advanced Computer Science and Applications, Vol. 4, No. 5, 2013.

[5] P. R. Sundaramoorthy, E. Gill, and C. J. M. Verhoeven, "Systematic Identification of Applications for a Cluster of Femto-satellites", 61th International Astronautical Congress, Prague, CZ,2010, pp 1-5.

[6] Geoffrey. R. McVittie. and Krishna Dev Kumar, "Design of a COTS Femtosatellite and Mission", AIAA Space 2007 Conference and Exposition.

[7] Joshua Tristancho, "Implementation of a Femtosatellite and a Mini-launcher", Msc Thesis, University of Polytechnic de Catalunya, 2010.

[8] Enric Fernandez-Murcia, Luis Izquierdo and Joshua Trishtancho," A Synthetic Aperture Antenna for Femtosatellites based on Commercial-of-the-shelf", IEEE 2011.

[9] A. R. Morse, "MSFC Skylab Apollo Telescope Mount", NASA Technical Memorandum, NASA TM X-64811, 1974.

[10] Lyndo, B., "Lessions Learned on the Skylab Program", NASA Technical Memorandum, NASA TM X-72920, 1974.

[11] Roser, X., and Sghedoni, M., "Control Moment Gyroscopes and Their Application in Future Scientific Missions", Proceeding 3rd International Conference on Spacecraft Guidance, Navigation and Control Systems, 1997, pp 523-528.

[12] Defindini, A., Faucheux, P., Guay, P., Bangert, K., Heimel, H., Privat, M., and Seiler, R., "Control Moment Gyro CMG 15-45s: A Compact CMG Product for Agile Satellites in the One Ton Class", Proceeding of the 10th European Space Mechanisms and Tribology Symposium, 2003, pp 27-31.

[13] Peck, M. A., Paluszek, M. A., Thomas, S. J. and Mueller, J. B., "Control Moment Gyroscopes for Joint Actuation: A New Paradigm in Space Robotics", AIAA 1st Space Exploration Conference: Continuing Voyage Discovery, 2005, pp 2005-2522.

[14] Thornton, B., Ura, T., and Nose, Y., "Combined Energy Storage and Three Axis Attitude Control of a Gyroscopically Actuated AUV", Proceeding of Oceans MTS/IEEE, 2008, pp 1-9.

[15] Craig Clark, Kevin Worral, Emre Yavuzoglu, "A Control Moment Gyro for Dynamic Attitude Control of Small Satellites", 24th Annual AIAA/USU Conference on Small Satellite Conference 2010, SSC10-XI-8,

[16] Mohammed Arezki Si Mohammed, "Simulation of Three Axis Attitude Control using a Control Momentum Gyroscope for Small Satellites", Proccedings of the World Congress on Engineering 2012, Vol II, WCD 2013, July 4-6, 2012, London, UK.

[17] Honglong Chang, Wenlong Jiao, Qianyan Fu, Jianbing Xie and Weizhang Yuan, "Design and Simulation of a 
MEMS Control Moment Gyroscope for the sub kilogram Spacecraft", Sensor, Vol. 10, pp 4130-4144, 2010.

[18] Joel Reiter, Karl Bohringer and Mark Campbell, "MEMS Control Moment Gyroscope Design and Wafter-based Spacecraft Chassis Study", In Proceedings of the SPIE Conference on Micromachined Devices and Components, Santa Clara, CA, USA, September 20-21, 1999, pp 122-128.

[19] D. J. Bell, T. J. Lu, N. A. Fleck, and S. M. Spearing, "MEMS Actuators and Sensors: Observations on their Performance and Selection for Purpose", Journal of Micromechanics and Microengineering, Vol. 15, 2005, pp 153-164.

[20] Y. L. Kuo, K. D. Kumar, K. Behdinan, and Z. Fawaz, ” Attitude Control of Miniature Spacecraft using MEMS Actutors", In Proceeding of Canadian Conferece on Electrical and Computer Engieering, Ottawa, ON, Canada, April 22-26, 2007, pp 160-163.

[21] D. J. Barnhart, "Very Small Satellite Design for Distributed Space Missions", Journal of Spacecraft and Rockets, 2007, Vol. 44, pp 1294-1396.

[22] W. C. Tang, "Micromechanical Devices at JPL for Space Exploration", IEEE Aerospace Conference, Snowmass at Aspen, CO, USA, March 21-28, 1998.

[23] Tony, K. Tang, Roman, C. Gutierrez, Christopher, B. Stell, Vatche Vorperian, Genji, A. Arakaki, John, Rice, Wen, J. Li, Indrani, Chakraborty, Kirill Sheheglov, Jaroslava, Z. Wileox, "A Packaged Silicon MEMS Vibratory Gyroscpe For Microspacecraft", Micro Electro Mechanical Systems, IEEE 10th Annual Internaitonal Workshop, Hotel Nagoya Castle, Japan, 1997.

[24] Wen-Ming Zhang, Han Yan, Zhi-ke Peng and Guang Meng, "Electrostatic Pull-in Instability in MEMS/NEMS: A Review", Sensors and Actuators A: Physical, 2014, pp 187-218.

[25] Gary, K. Maki and Pen-shu Yeh, "Radiation Tolerant Ultra Power CMOS Microelectronics Technology Development Status", NASA Earth Science Technology Conference 2003, June 24-26, University of Maryland Inn and Conference Center.

[26] Joel Gambino and Neil Dennehy, "A Multi Function Guidance, Navigatin and Control System for Future Earth and Space Science Missions", 25th Annual AAS Guidance and Control Conference, February 6-10, 2002, Breackenridge, Colorado.

[27] Jean-Francois Saheb, Jean-Francois Richard, R. Meingan, M. Sawan, and Y. Savaria, "System Integration of High Voltage Electrostatic MEMS Actuators", IEEE 2005.

[28] Memscap Inc., Research Triangle Park, NC, USA. 12 Alexander Drive, Building 100. [Online]. Available: http://www.memscapinc.com

[29] J. Andrew Yeh, Chi-Nung Chen, and Yen-Seng Lui, "Large Rotation Actuated by in plane Rotary Combdrives with Serpentine Spring Suspension", Journal of Micro-mechanics and Microengineering, Vol. 15, 2005, pp 201-206.

[30] Honglong Chang, Bin Bai, Wenlong Jiao, and Jianbing Xie, "Design of a Micro Control Moment Gyroscope with a Large Torque Output" (in Chinese), Chinese Journal of Sensors and Actuators, Vol. 24, No. 8, 2011.

[31] M. A. Hopcroft, W. D. Nix, and T. W. Kenny, "What is the Young's Modulus of Silicon?", IEEE Journal of
Microelectromechanical Systems, 2010, vol. 19, pp. 229-238.

[32] R. Bauer, A. Paterson, C. Clark, D. Uttamchandani, and W. Lubeigt, "Output Characteristics of Q-switched Solid-State Lasers Using Intracavity MEMS Micromirrors", IEEE Journal of Selected Topics in Quantum Electronics, 2015, vol. 21, pp. 356-363.

[33] Eldwin Jiaqiang Ng, Vu Hong, Yi Yang, Chong $\mathrm{H}$. Ahn, Camille LM Everhart, and Thomas W. Kenny, "Temperature Dependence of the Elastic Constants of Doped Silicon," in Journal of Microelectromechanical Systems, vol.24, no.3, June 2015, pp.730-741

[34] Guangjun Liu, Feng Yang, Xiaofan Bao, and Tao Jiang. "Robust Optimization of a MEMS Accelerometer Considering Temperature Variations." Sensors 15, no. 3, 2015, pp 6342-6359.

[35] Shirin Ghaffari, Eldwin Jiaqing Ng, Chae Hyuck Ahn, Yushi Yang, Shasha Wang, Vu A. Hong, and Thomas W. Kenny, "Accurate Modeling of Quality Factor Behavior of Complex Silicon MEMS Resonators", Journal of Microelectromechanical Systems, Vol. 24, No.2, 2015, pp 276-288.

[36] Igor P. Prikhodko, Alexander A. Trusov and Andrei M. Shkel, "Compensation of Drifts in High Q MEMS Gyroscopes using Temperature Self-sensing", Sensors and Actuators A: Physical, Vol 201, 2013, pp 517-524.

[37] T. F. Tan and K. Weber and C. K. H. Dharan, "Failure Analysis of Thermal Actuators, Comb Drives, and Other Microelectromechanical Elements", Journal of Failure Analysis and Prevention, April 2007, Vol. 7, No. 2, pp 137-143.

[38] Herbert R. Shea, "Reliability of MEMS for space applications", in "Moems-mems 2006 micro and nanofabrication", International Society for Optics and Photonics, 2006, pp 61110A-61110A.

[39] Herbert R. Shea, "Effects of radiation on MEMS", in "SPIE MOEMS-MEMS", International Society for Optics and Photonics, 2011, pp 79280E-79280E.

[40] A. Lozano and F. Palumbo and F. Alurralde, "Radiation Effects on SOI Electrostatic Comb Drive Actuators of MEMS Devices", Proceedings of the Argentine School of Micro-Nanoelectronics, Technology and Applications, 2009, pp 46-49.

\section{BIOGRAPHY}

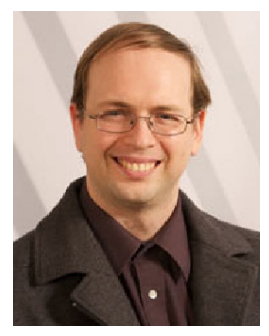

Mark Post received his B.A.Sc. degree in electrical engineering from the University of Toronto in 2004 and his M.Sc. and Ph. D. degrees in Space Science and Engineering from York University, Canada in 2008 and 2014. He currently holds the position of lecturer at the University of Strathclyde in the United Kingdom. His research includes mechatronic and embedded design of reliable and efficient robotic space systems and intelligent algorithms for autonomous control for spacecraft and planetary rovers. 


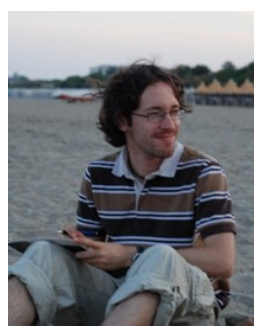

Ralf Bauer received his Dipl.-Ing. degree in Mechatronics from the University of Erlangen-Nuernberg, Germany in 2010 and his Ph.D. degree in the Department of Electronic and Electrical Engineering from the University of Strathclyde, U.K. in 2013. He is currently a Research Associate at the University of Strathclyde in the United Kingdom. His research interests are MEMS enabled miniaturisation of biomedical devices, optical sensors and laser systems.

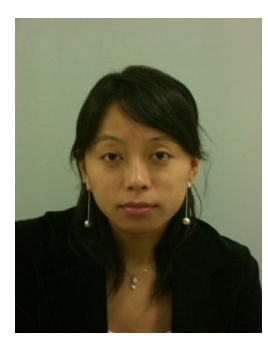

Junquan $\mathrm{Li}$ received her M.sc. from the University of Science and Technology Beijing, China in 2006 and Ph.D. in Department of Aerospace Engineering from Ryerson University, Canada in 2012. She was a MITACS PDF fellow in Canada during 2012-2013. She is a Marie-Curie PDF researcher at Astronet II FP7 networks in the United Kingdom from 2014-2015. Her research areas are attitude and orbit determination and control, nanosatellite and CubeSat engineering, formation flying, and fault tolerant control theory and applications.

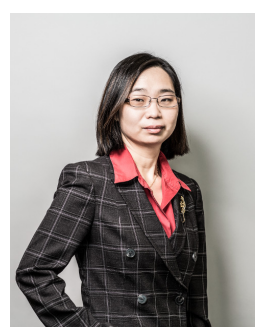

Regina Lee received her B.A.Sc in 1994 and M.A.Sc in 1995 from the University of Toronto. She received her Ph.D. from the University of Toronto in 2000. She is currently an associate professor and chair of the department of Earth and Space Science Engineering at York University Canada. Prof. Lee' research interests center on nanosatellite technology development, micro propulsion system design, MEMS based attitude sensor, actuator design and algorithms and FPGA subsystem development. 